\title{
Flexible ACT \& Resource-group ACT: Different Working Procedures Which Can Supplement and Strengthen Each Other. A Response
}

\author{
Remmers van Veldhuizen ${ }^{1, *}$, Philippe Delespaul ${ }^{2}$, Hans Kroon $^{3}$ and Niels Mulder ${ }^{4}$
}

${ }^{1}$ CCAF (Certification Centre for ACT and FACT), Utrecht, The Netherlands; ${ }^{2}$ Innovations in Mental Health Care, Maastricht University and Mondriaan (Mental Health Service), The Netherlands; ${ }^{3}$ Reintegration and Community Care, Trimbos Institute, Utrecht, The Netherlands; ${ }^{4}$ Public Mental Health, Epidemiological and Social Psychiatric Research institute, Erasmus MC; Parnassia Psychiatric Institute, The Netherlands

\begin{abstract}
This article is a response to Nordén and Norlander's 'Absence of Positive Results for Flexible Assertive Community Treatment. What is the next approach?'[1], in which they assert that 'at present [there is] no evidence for Flexible ACT and... that RACT might be able to provide new impulses and new vitality to the treatment mode of ACT'. We question their analyses and conclusions. We clarify Flexible ACT, referring to the Flexible Assertive Community Treatment Manual (van Veldhuizen, 2013) [2] to rectify misconceptions. We discuss Nordén and Norlander's interpretation of research on Flexible ACT. The fact that too little research has been done and that there are insufficient positive results cannot serve as a reason to propagate RACT. However, the Resource Group method does provide inspiration for working with clients to involve their networks more effectively in Flexible ACT.
\end{abstract}

Keywords: ACT, community care, FACT, flexible ACT, RACT, resource ACT.

\section{INTRODUCTION}

In their article, Nordén and Norlander [1] describe Flexible ACT as it was presented in 2007 (van Veldhuizen, 2007) [2]. Unfortunately the authors did not consult the more upto-date Flexible ACT Manual (van Veldhuizen \& Bähler, 2013) [3], which was presented at the second conference of the European Assertive Outreach Foundation in 2013 and then brought to international attention through email and websites (downloadable free of charge in English, French, Norwegian and Swedish at www.factfacts.nl).

Flexible ACT is a service delivery model that aims to provide the best possible treatment and support for SMI patients. Flexible ACT is continually enriched by new insights, partly through related models such as RACT. For example, Falloon's integrated mental health care model (1998) [4] inspired Dutch pioneers in South Limburg, who first called their care innovation for SMI patients 'integrated care', but later concurred with the name FACT.

We will point out a few developments between 2007 and 2013. The 2007 article still refers to a FACT whiteboard, but in 2008 the digital FACT board was introduced. In 2008 the Dutch version of the FACT manual (van Veldhuizen et al.) appeared and in 2010 the second version of the FACT scale (FACTs), which the Centre for Certification of ACT and

*Address correspondence to this author at the CCAF, PO Box 543, 3440 AM Woerden, The Netherlands, Tel: +31653233193 ;

E-mail: remmersvv@hotmail.com

\#A commentary article in response to 'Absence of Positive Results for Flexible Assertive Community Treatment. What is the next approach?' (T. Nordén \& T. Norlander in Clinical Practice \& Epidemiology in Mental Health, 2014: 10, 87-91.)
FACT (CCAF) has now used to certify over 200 teams. In 2014 FACT has moved further away from stressing damage limitation towards focusing on maximising the resilience of people with SMI in their own environments. Flexible ACT has developed strongly in the direction of recovery-oriented care. Most FACT teams now include a peer support worker. There is also a stronger emphasis on social inclusion, in which the client's family is obviously involved. The Flexible ACT Manual discusses the importance of the Strengths Model, recovery and rehabilitation. The focus is on goals clients themselves formulate for their housing, work and relationships in the community. As well as the case manager, often the IPS job coach and the peer support worker in the Flexible ACT team are involved. Evidence-based practices are used.

\section{RESPONSE REGARDING THE FLEXIBLE ACT MODEL}

Nordén and Norlander quote from van Veldhuizen (2007), referring to an individual treatment plan 'which is renewed at least once a year and is formulated in ways that the patients and families can understand'. They regard this as a 'more orthodox model'. In their view, the fact that in their approach the starting point is the client's needs and wishes is 'the decisive difference between RACT and other ACT'. However, we disagree with this. In Flexible ACT the treatment plan is drawn up on the basis of wishes formulated by the client and of the needs and priority areas noted in Routine Outcome Monitoring. The client's family is involved in this process, in consultation with the client. Nordén and Norlander criticise the phrase 'formulated in a way that patients and their families can understand'. This criticism is also unjustified. A good treatment plan is written in clear, personal 
language, with the client's own treatment goals written in the first person. In other words, our intentions and practice have for years been identical to what RACT now claims as a unique feature.

In the sentence quoted, we say that we evaluate and renew the treatment plan 'at least' once a year (in conjunction with the client and their family or friends). But this may happen more often. In teams which work optimally according to the model, the treatment plan (and the crisis detection and intervention plan that goes with it) is evaluated and if necessary modified whenever the client is placed on the FACT board or taken off it for any reason whatsoever. The team then talks to the client and family about how well intensive care with shared case load has worked. Interventions which have proved effective can be included in the crisis plan. This evaluation also looks at what the family and close friends can do and how heavy the burden is for them.

Nordén and Norlander mistakenly assert that Flexible ACT (along with ACT and AOT) is 'surprisingly enough not focused on providing service and treatment to the majority of clients who are largely in a stable phase'. This is plainly incorrect. Flexible ACT's basic vision is that care for this stable group and care for the instable group should be combined in one team. The reason why this is so important is because clients' needs change due to remission and relapse, crisis and recovery; sometimes they need very intensive care, similar to ACT. At other times they are much more in need of rehabilitation and recovery support, optimized medication regimes or psychotherapy. For the sake of continuity of care and of treating practitioners, Flexible ACT combines these two types of care in one team. The 'stable' clients receive support and treatment based on their treatment plan, coordinated by their case managers. Guided by the client's needs and problems, the multidisciplinary team also provides contact with a psychiatrist, a psychologist, a peer support worker, an addiction specialist or a job coach as required. These contacts are coordinated by the case manager in consultation with the client (and their network). At least once a year the client often accompanied by the case manager - talks to the psychiatrist about issues such as medication, physical screening and wishes regarding the treatment plan. In other words, 'stable clients' see their case manager at changing intervals sometimes weekly, sometimes once every 2-4 weeks - and usually also 2-4 other team members at widely varying intervals. A client may be having EMDR trauma therapy from the psychologist or may be looking for a job with the help of a job coach. The client may also be in a recovery group set up by the peer support worker. In practice, 'stable' clients do not see the whole Flexible ACT team, but work with their own 'sub-team' of 2 to 5 team members. Although this subteam has no separate status in the Flexible ACT model, we see close similarities between this system and the availability of practitioners in RACT's resource group in. The difference lies in the participation of other (non-professional) people (family, friends and others from the client's external support system) in a resource group and in different arrangements for guidance and management.

We therefore distance ourselves from Nordén and Norlander's incomplete and partly incorrect description of some aspects of the Flexible ACT model. The lesson for us is that we should be cautious about judging each other's pro- grammes and everyday practices in different international contexts.

\section{RESPONSE REGARDING RESEARCH ON FLEXI- BLE ACT}

Nordén and Norlander assert that there is insufficient evidence for the effectiveness of Flexible ACT. We agree. Although the Flexible ACT model has been implemented on a large scale in the Netherlands, the developers of Flexible ACT have not managed to organize an RCT of the effectiveness of Flexible ACT. After 2008 this proved to be unfeasible, because facilities were no longer willing to provide control groups receiving 'care as usual'. They opted for Flexible ACT as a service delivery model which enabled them to ensure that evidence-based interventions in line with the Dutch Multidisciplinary Guideline for Schizophrenia could be provided in the client's own environment. In the Netherlands there was no other model available which could provide care for the whole group of people with schizophrenia or some other SMI which was consistent with the recommendations of the Guideline and could incorporate recovery-oriented care. The rapid implementation of the model stood in the way of research.

Nevertheless, there has been some research [5-8]. And we disagree with Nordén and Norlander when they seem to imply that all the results were null findings. The authors of the original articles pointed out the limitations of their research - as is correct procedure - but they did find some positive trends. Only one of the studies reported a null finding. Moreover, they ignored the study of the relationship between model fidelity and outcomes (van Vugt, 2011)[9]. Research among 20 ACT and FACT teams shows favourable connections between model fidelity and outcomes such as general functioning and homelessness. Van Vugt et al. found evidence that team organization (shared caseload) was an important element. Teams with peer support workers had better effects. Higher model fidelity did not predict better results as regards number of admission days.

To draw valid conclusions about FACT and RACT, Nordén and Norlander would have to discuss the quality of all 17 studies of RACT as well as the 5 studies of FACT. More importantly, however, none of the studies included in the meta-analysis by Nordén et al. (2012)[10] examined RACT as a whole, but only one or more elements of it. A new review which examined the 17 studies of RACT and the 5 of FACT might well conclude that as regards certain elements RACT is more effective than FACT. However, the populations covered by RACT and FACT seem to differ (severity of symptoms, diagnoses), which means that even given the limitations of the FACT studies, it would still be impossible to conclude that RACT is more effective than FACT. To make a valid comparison between the two, Nordén and Norlander would need studies that assess the effectiveness of RACT and FACT in the same population, and as far as we know there are no such studies. Moreover, we do not believe a comparison of RACT and FACT would be helpful, because FACT is a service delivery model, whereas RACT is a separate intervention. However, RACT could be integrated into the FACT model to assess whether or not clients benefit from the addition of this intervention. 
Obviously, all of the Dutch studies of FACT focus on FACT populations. Nordén and Norlander erroneously identify this as a limitation. They suggest stratifying ACTeligible clients and non-ACT-eligible clients in future research. However, ACT for ACT-eligible clients has been widely studied, whereas FACT for non-ACT-eligible clients has never been studied separately. This is a possibility for further research, but not a limitation.

Nordén and Norlander also discuss the UK-based study by Firn et al. [11]. We cannot help feeling that once again their conclusion is overly pessimistic. In particular, they disregard the significant positive findings concerning reduced service utilization by clients after the closure of ACT teams in the UK dismantling study (Firn et al.) and instead highlight model fidelity issues and a non-significant employment outcome. The dismantling study is an evaluation of service delivery in real-life situations, with practical applications in struggling healthcare economies. It was not a rigorous trial and some criticism may be due, but the central findings are unquestionably significant.

In summary, Nordén and Norlander are right in saying there is as yet insufficient evidence for Flexible ACT, but this is mainly because very little research has been done on it. Moreover, in our opinion they have paid too little attention to the positive results that have emerged.

New studies are now underway both in the UK and in the Netherlands. In the north-west of the Netherlands a cohort study has begun of Flexible ACT implementation in three teams. Outcomes include care consumption (admissions, admission days, outpatient face-to-face contacts), clinical outcomes (care needs, quality of life, functioning), social inclusion indicators (social contacts, employment and housing situation) and satisfaction with care. The level of implementation of Flexible ACT was also assessed. This study is now under review. The preliminary results were presented at the EAOF Congress in Aviles, Spain, in 2013. These results were positive as regards care consumption, care needs and quality of life. This study and the studies by Drukker and Bak [5-8] rightly pay attention to client-oriented results and the satisfaction of clients and their families for the care provided, whereas formerly the emphasis was often on reducing admission days. We hope that in countries where interest has been shown in Flexible ACT (such as the UK, Belgium, Canada, Norway and Sweden) comparative research can be organized and possibly RCTs which focus on client-oriented outcomes.

\section{RACT}

Nordén and Norlander do not provide a comprehensive account of RACT as a model in their article. From the article they quote from (Nordén et al.) [10] we understand that for each client a 'resource group' is formed that consists not only of care practitioners (case manager and psychiatrist), but also of the clients themselves, family members, neighbours or other people who are involved with the client in the community. In this group clients determine their own goals and remain in control, supported by the case manager. As set out above, Flexible ACT already works intensively with families and other informal carers. Sometimes this is very successful, but a great deal still needs to be developed as regards participation and inclusion in the community. In the Netherlands the 'welfare state' paradigm is developing towards a 'participation society'. This means we must draw on clients' own strengths and those of their networks and work with non-professional support factors in society. In the Netherlands a study is underway of a decision-making model known as Family Group Conferences (FGCs). At an FGC everyone who is involved with the client and able to contribute is invited. Not only the client, but also their family and carers are present, and friends and neighbours can also attend. A key feature of the model is the private time during which professionals leave the meeting and the client and their network develop their own plan. This empowers clients, giving them the responsibility for improving their own lives. For mental health services FGCs may be a way to create partnerships with clients and communities (de Jong et al., 2014)[12].

The 'resource group' in RACT seems to work in a similar way, though there are differences. The group is not incidentally involved, but more long-term and systematically. In addition, RACT gives the care practitioners a more significant role in the group. We see the systematic involvement of the client's personal network and external support services as a possible enrichment of Flexible ACT teams. On the other hand, we question whether RACT makes enough use of outreach care. With Flexible ACT we have found that outreach (both for instable and stable clients and their networks) is a decisive factor in helping clients to achieve their goals in the environment in which they want to succeed. In the Netherlands four Flexible ACT teams have now added RACT. The two models seem to be quite compatible and to enrich each other, with Flexible ACT as the service delivery model (for both stable and instable clients), while RACT provides a specific contribution to formulating and working with others to achieve the client's treatment and social inclusion goals.

\section{CONCLUSION}

One model is not automatically better because another model has been insufficiently studied. Thorough research on Flexible ACT is needed. Perhaps other countries can arrange an RCT of Flexible ACT compared with care as usual, possibly with the addition of RACT interventions.

We distance ourselves from Nordén and Norlander when they outline some aspects of Flexible ACT in a negative way. We stress that in Flexible ACT treatment and support are shaped by a treatment plan based on the client's wishes and needs, in consultation with the client and family. Stable clients have comprehensive programmes in which they work with a small group of professionals and which also involve the family. For a more detailed account of the Flexible ACT model we refer to the Manual (van Veldhuizen, 2013). We conclude that the interventions developed in RACT fit in well with Flexible ACT and may improve clients' social inclusion. There is every reason for us to collaborate. If our goal is to empower clients and support their recovery, we have an obligation as treating practitioners and researchers to combine our resources.

\section{CONFLICT OF INTEREST}

The authors confirm that this article content has no conflict of interest. 


\section{ACKNOWLEDGEMENTS}

Declared none.

\section{REFERENCES}

[1] Nordén T, Norlander T. Absence of positive results for flexible assertive community treatment. What is the next approach? Clin Pract Epidemiol Ment Health 2014: 10: 87-91.

[2] Van Veldhuizen JR. FACT: a Dutch version of ACT. Community Ment Health J 2007; 43: 421-33.

[3] Van Veldhuizen JR, Bähler M. Manual flexible assertive community treatment. The Netherlands: FACT manual, Groningen 2013.

[4] Falloon IRH, Fadden G. Integrated Mental Health Care. Cambridge: Cambridge University Press 1993.

[5] Bak M, van Os J, Delespaul P, et al. An observational, 'real life' trial of the introduction of assertive community treatment in a geographically defined area using clinical rather than service use outcome criteria. Soc Psychiatry Psychiatr Epidemiol 2007; 42: 125 30 .

[6] Drukker M, Maarschalkerweerd M, Bak M, et al. A real-life observational study of the effectiveness of FACT in a Dutch mental health region. BMC Psychiatry 2008; 8: 93.
[7] Drukker M, van Os J, Sytema S, Driessen G, Visser E, Delespaul P. Function assertive community treatment (FACT) and psychiatric service use in patients diagnosed with severe mental illness. Epidemiol Psychiatr Sci 2011; 20: 273-8.

[8] Drukker M, Visser E, Sytema S, van Os J. Flexible assertive community treatment: severity of symptoms and psychiatric health service use, a real life observational study. Clin Pract Epidemio Ment Health 2013; 9: 202-9.

[9] Van Vugt MD, Kroon H, Delespaul PH, et al. Assertive community treatment in The Netherlands: outcome and model fidelity. Can J Psychiatry 2011; 56: 154-60.

[10] Nordén T, Eriksson A, Kjellgren A, Norlander T. Involving clients and their relatives and friends in psychiatric care: Case managers' experiences of training in resource group assertive community treatment. Psych J 2012; 1: 15-27.

[11] Firn M, Hindhaugh K, Hubbeling D, Davies G, Jones B, White SJ A dismantling study of assertive outreach services: comparing activity and outcomes following replacement with the FACT model Soc Psychiatry Psychiatr Epidemiol 2013; 48: 997-1003.

[12] De Jong G, Schout G, Abma T. Prevention of involuntary admission through family group conferencing: a qualitative case study in community mental health nursing. J Adv Nurs 2014; 70(11): 265162 .

Received: December 18, 2014

Revised: December 22, 2014

Accepted: December 22, 2014

(C) Veldhuizen et al.; Licensee Bentham Open.

This is an open access article licensed under the terms of the Creative Commons Attribution Non-Commercial License (http://creativecommons.org/licenses/by-nc/3.0/) which permits unrestricted, non-commercial use, distribution and reproduction in any medium, provided the work is properly cited. 